\title{
PROJECT ARCHES: \\ AN EVALUATION OF A MODIFIED FAMILY CHECK-UP INTERVENTION \\ IN AN ASSESSMENT SETTING
}

\author{
Lindsay A. Borden \\ Dr. Keith C. Herman, Dissertation Supervisor
}

\begin{abstract}
Providing feedback to parents after child psychological evaluations is a critical opportunity for mental health professionals to directly impact problem recognition, treatment attendance and engagement, and subsequent outcomes. However, research examining evidencebased and therapeutic forms of feedback is limited and current frameworks largely neglect the importance of addressing family engagement and motivation during feedback sessions. The purpose of the present study was threefold: 1) to develop a family-focused assessment feedback process using principles from the evidence-based Family Check-Up intervention and motivational interviewing (MI), 2) to train student clinicians to implement feedback sessions, and 3 ) to evaluate both feedback and training procedures. Participants included 5 graduate student clinicians and 10 parent-child client dyads recruited from a university-based clinic that provides comprehensive evaluations to children in a Midwestern community. Both clinicians and parents expressed positive perceptions of the feedback sessions. Clinicians' perceived MI self-efficacy increased after training sessions and objective coding of feedback sessions revealed use of greater MI-consistent than inconsistent language that resulted in subsequent parent change talk. Limitations and implications for future research are discussed.
\end{abstract}

